\title{
Central limit theorem for Fourier transform and periodogram of random fields
}

\author{
MAGDA PELIGRAD* and NA ZHANG ${ }^{* * *}$ \\ Department of Mathematical Sciences, University of Cincinnati, PO Box 210025, Cincinnati, OH 45221- \\ 0025, USA.E-mail: *peligrm@ucmail.uc.edu; ${ }^{* *}$ zhangn4@mail.uc.edu
}

In this paper, we show that the limiting distribution of the real and the imaginary part of the Fourier transform of a stationary random field is almost surely an independent vector with Gaussian marginal distributions, whose variance is, up to a constant, the field's spectral density. The dependence structure of the random field is general and we do not impose any restrictions on the speed of convergence to zero of the covariances, or smoothness of the spectral density. The only condition required is that the variables are adapted to a commuting filtration and are regular in some sense. The results go beyond the Bernoulli fields and apply to both short range and long range dependence. They can be easily applied to derive the asymptotic behavior of the periodogram associated to the random field. The method of proof is based on new probabilistic methods involving martingale approximations and also on borrowed and new tools from harmonic analysis. Several examples to linear, Volterra and Gaussian random fields will be presented.

Keywords: central limit theorem; Fourier transform; martingale approximation; random field; spectral density

\section{Introduction}

The discrete Fourier transform, defined as

$$
S_{n}(t)=\sum_{k=1}^{n} \mathrm{e}^{\mathrm{i} k t} X_{k},
$$

where $\mathrm{i}=\sqrt{-1}$ is the imaginary unit, plays an essential role in the study of stationary time series $\left(X_{j}\right)_{j \in Z}$ of centered random variables with finite second moment, adapted to a filtration $\left(\mathcal{F}_{u}\right)_{u \in Z}$.

The periodogram, introduced as a tool by Schuster [27], is essential for the estimation of the spectral density of the stationary processes. It is defined by

$$
I_{n}(t)=\frac{1}{2 \pi n}\left|S_{n}(t)\right|^{2}, \quad t \in[-\pi, \pi) .
$$

There is a vast literature concerning these statistics. They are often used to determine hidden periodicities. Denote by $\lambda$ the Lebesgue measure on the real line. In Peligrad and Wu [24], it was proved a surprising result, that, under ergodicity and a very mild regularity condition, for $\lambda$-almost all frequencies $t$, the random variables $\operatorname{Re} S_{n}(t) / \sqrt{n}$ and $\operatorname{Im} S_{n}(t) / \sqrt{n}$ are asymptotically independent identically distributed random variables with normal distribution, mean 0 and variance $\pi f(t)$. Here $f$ is the spectral density of $\left(X_{j}\right)_{j \in Z}$. The regularity condition, namely 
$E\left(X_{0} \mid \mathcal{F}_{-\infty}\right)=0$ a.s., is a mild restriction of dependence and accommodates large classes of sequences with short and long range dependence. This result implies that for $\lambda$-almost all $t$, the periodogram $I_{n}(t)$ converges in distribution to $f(t) \chi^{2}$, where $\chi^{2}$ has a chi-square distribution with 2 degrees of freedom, even in the case of processes with long memory. The proof of this result is based on the celebrated Carleson theorem [4] about almost sure convergence of Fourier transforms, combined with martingale approximations and Fourier analysis.

In this paper, we analyze the asymptotic properties of the Fourier transform for random fields. Let $d$ be a positive integer. We start with a strictly stationary random field $\left(X_{\mathbf{u}}\right)_{\mathbf{u} \in Z^{d}}$ of square integrable and centered random variables. We introduce the discrete Fourier transform for random fields by the rotated sum

$$
S_{\mathbf{n}}(\mathbf{t})=\sum_{\mathbf{1} \leq \mathbf{u} \leq \mathbf{n}} \mathrm{e}^{\mathrm{iu} \cdot \mathbf{t}} X_{\mathbf{u}},
$$

where we have $\mathbf{1} \leq \mathbf{u} \leq \mathbf{n}$ and $\mathbf{t} \in I=[-\pi, \pi)^{d}$. By $\mathbf{u} \leq \mathbf{n}$ we understand $\mathbf{u}=\left(u_{1}, \ldots, u_{d}\right)$, $\mathbf{n}=\left(n_{1}, \ldots, n_{d}\right)$ and $1 \leq u_{1} \leq n_{1}, \ldots, 1 \leq u_{d} \leq n_{d}$. Also $\mathbf{u} \cdot \mathbf{t}=u_{1} t_{1}+\cdots+u_{d} t_{d}$.

For a weakly stationary random field the spectral analysis was initiated in several papers by Helson and Lowdenslager [15], Kallianpur et al. [20] and Francos et al. [11]. These papers stress the huge difficulties when one tries to extend the results from sequences of random variables to random fields. One of the difficulty is that for random fields the future and past do not have a unique interpretation. Also, many of the important spectral analysis results relevant to the proofs, do not fully extend to double indexed sequences, including the celebrated Fejér-Lebesgue theorem (cf. Bary [1], page 139) or the Carleson theorem [4] (see Fefferman [10]). To compensate for the lack of ordering of the filtration, we utilize the notion of commuting filtration. Such filtrations have a certain Markovian character. For instance, for $d=2$, we can start with a stationary random field with independent rows or columns which generate a commuting filtration. Then, we construct a stationary random field which is a function of the initial one.

The main result of the paper is a natural extension from sequences of random variables, indexed by integers, to random fields of the result of Peligrad and $\mathrm{Wu}$ [24]. Under certain regularity conditions, we shall prove that, almost surely in $\mathbf{t} \in I$, both the real and imaginary part of $S_{\mathbf{n}}(\mathbf{t}) / \sqrt{n_{1} \cdots n_{d}}$ converge to independent normal variables whose variance is, up to a multiplicative constant, the spectral density of the random field, denoted by $f(\mathbf{t})$. The ergodicity condition is imposed to only one of the directions of the random field.

The periodogram, has the following extension to the random field:

$$
I_{\mathbf{n}}(\mathbf{t})=\frac{1}{(2 \pi)^{d} n_{1} \cdots n_{d}}\left|\sum_{\mathbf{1} \leq \mathbf{u} \leq \mathbf{n}} \mathrm{e}^{\mathrm{iu} \cdot \mathbf{t}} X_{\mathbf{u}}\right|^{2}, \quad \mathbf{t} \in I .
$$

Our result is that, for almost all frequencies $\mathbf{t} \in I$, the limiting distribution of $I_{\mathbf{n}}(\mathbf{t})$ is $f(\mathbf{t}) \chi^{2}(2)$, where $\chi^{2}(2)$ is a chi-square distribution with two degrees of freedom.

The proof is based on a new, interesting representation for the spectral density in terms of projection operators, which is the most important tool for establishing our result. The proof also involves a martingale approximation for random fields as well as laws of large numbers for Fourier sums, which have interest in themselves.

We consider two types of summations. The first result is for summations of the variables in a multi-dimensional cube. The reason we first restrict ourselves to summations indexed by 
the cubes is due to the relation between our results and optimal results available in harmonic analysis. For example, for $d=2$, Theorem 1 in Marcinkiewicz and Zygmund [22] shows that the Fejér-Lebesgue theorem holds for spectral densities in $L_{1}$ when the summation is taken over rectangles of size $m \times n$, provided that $m, n \rightarrow \infty$ such that $m / n \leq a$ and $n / m \leq a$ for some positive number $a$. This result fails when the summation is taken over general rectangles. However, if the summation is taken over the sets $1 \leq u_{1} \leq n, 1 \leq u_{2} \leq m$, where $n \geq m \rightarrow \infty$, one should assume the integrability of $f(\mathbf{u}) \ln ^{+} f(\mathbf{u})$ as a minimal condition for the validity of the Fejér-Lebesgue theorem (see Jessen et al. [19]). We shall also give a result in this context, where the summation is taken over unrestricted rectangles.

When dealing with random fields the notation can become rather complicated. This is the reason why, for presenting the material, we implemented the following strategy: We treat first the case $d=2$. Then, we mention the small differences for treating the general case of multidimensional index set by using the mathematical induction.

Our paper is organized as follows. In a preliminary section, we review several facts about limiting variance of the Fourier series, introduce the notions of stationary random fields and commuting filtrations. In Section 3, we obtain a representation of the spectral density in terms of projection operators, which extends a recent result by Lifshitz and Peligrad [21] beyond the setting of Bernoulli shifts. We also state and prove our main results on the limiting distribution of double indexed, random Fourier sums. The extension to general index set is given in Section 4 . Section 5 is dedicated to examples, such as functions of Gaussian sequences, linear and nonlinear random fields with independent innovations. It is remarkable that the only condition required for the validity of our results for linear or Volterra random fields with independent innovations is equivalent to merely the existence of these fields. In a supplementary section, we prove two laws of large numbers and other lemmas about commuting filtrations.

Our paper joins the recent increased interest in finding martingale methods for random fields, initiated by Rosenblatt [26] and continued by Gordin [14]. We would like to mention several remarkable papers in this direction. For instance, the paper by Volný and Wang [29] treated projection conditions and orthomartingales. Volný [28] discovered that the fields of stationary orthomartingales require the ergodicity in only one of the directions of the field as a necessary condition for the CLT. Cuny et al. [7] treated dynamical system via projection conditions. The paper of El Machkouri et al. [8] deals with random fields which are functions of i.i.d. Wang and Woodroofe [30], Peligrad and Zhang [25] and Giraudo [13] treated the Maxwell-Woodroofe condition. Also, in the context of dynamical systems, the CLT for Fourier transform for random fields was stated in Cohen and Conze [5] for $K$-systems. It should also be mentioned that a central limit theorem for periodogram of random fields was obtained by Miller [23] under mixing conditions. All these papers were inspirational.

\section{Preliminaries}

\section{Spectral density and limiting variance}

We call the complex valued zero mean field of random variables $\left(X_{\mathbf{m}}\right)_{\mathbf{m} \in Z^{2}}$ defined on a probability space $(\Omega, \mathcal{K}, P)$, weakly stationary (or second order stationary), if there are complex 
numbers $\gamma(\mathbf{m}), \mathbf{m} \in Z^{2}$, such that for all $\mathbf{u}, \mathbf{v} \in Z^{2}$,

$$
\operatorname{cov}\left(X_{\mathbf{u}}, X_{\mathbf{v}}\right)=E\left(X_{\mathbf{u}} \bar{X}_{\mathbf{v}}\right)=\gamma(\mathbf{u}-\mathbf{v}) .
$$

In the context of weakly stationary random fields, it is known that there exists a unique measure on $I=[-\pi, \pi)^{2}$, such that

$$
\gamma(\mathbf{u})=\int_{I} \mathrm{e}^{\mathrm{i} \mathbf{u} \cdot \mathbf{x}} F(\mathrm{~d} \mathbf{x}), \quad \text { for all } \mathbf{u} \in Z^{2},
$$

where $\mathbf{u} \cdot \mathbf{x}$ is the inner product. If $F$ is absolutely continuous with respect to Lebesgue measure $\lambda^{2}$ on $I=[-\pi, \pi)^{2}$ then, the Radon-Nikodym derivative $f$ of $F$ with respect to the Lebesgue measure is called spectral density $(F(\mathrm{~d} \mathbf{t})=f(\mathbf{t}) \mathrm{d} \mathbf{t})$, and we have

$$
\gamma(\mathbf{u})=\int_{I} \mathrm{e}^{\mathrm{i} \mathbf{u} \cdot \mathbf{x}} f(\mathbf{x}) \mathrm{d} \mathbf{x}, \quad \text { for all } \mathbf{u} \in Z^{2}
$$

The variance of partial sums on rectangles is

$$
E\left|S_{\mathbf{n}}(\mathbf{t})\right|^{2}=\sum_{\mathbf{1} \leq \mathbf{u}, \mathbf{v} \leq \mathbf{n}} \gamma(\mathbf{u}-\mathbf{v}) e^{\mathrm{it} \cdot(\mathbf{u}-\mathbf{v})} .
$$

Well-known computations show that

$$
\begin{aligned}
E\left|S_{\mathbf{n}}(\mathbf{t})\right|^{2} & =\sum_{\mathbf{1} \leq \mathbf{u}, \mathbf{v} \leq \mathbf{n}} e^{\mathrm{it} \cdot(\mathbf{u}-\mathbf{v})} \int_{I} e^{\mathrm{i} \mathbf{x} \cdot(\mathbf{u}-\mathbf{v})} f(\mathbf{x}) \mathrm{d} \mathbf{x} \\
& =\int_{I} \sum_{\mathbf{1} \leq \mathbf{u}, \mathbf{v} \leq \mathbf{n}} e^{\mathrm{i} \mathbf{x} \cdot(\mathbf{u}-\mathbf{v})} f(\mathbf{x}-\mathbf{t}) \mathrm{d} \mathbf{x} .
\end{aligned}
$$

So, with the notation $\mathbf{x}=\left(x_{1}, x_{2}\right)$, one can rewrite

$$
\frac{1}{n_{1} n_{2}} E\left|S_{\mathbf{n}}(\mathbf{t})\right|^{2}=\int_{I} K_{n_{1}}\left(x_{1}\right) K_{n_{2}}\left(x_{2}\right) f(\mathbf{x}-\mathbf{t}) \mathrm{d} \mathbf{x},
$$

where $K_{n}(x)$ is the Fejér Kernel

$$
K_{n}(x)=\sum_{|j|<n}\left(1-\frac{|j|}{n}\right) \mathrm{e}^{\mathrm{i} j x} .
$$

Furthermore, by Theorem 1 in Marcinkiewicz and Zygmund [22], for $\lambda^{2}$-almost all $\mathbf{t}$ in $I$, we obtain a limiting representation for the spectral density, namely

$$
\lim _{n \rightarrow \infty} \frac{1}{n^{2}} E\left|S_{n, n}(\mathbf{t})\right|^{2}=(2 \pi)^{2} f(\mathbf{t})
$$


If in addition $f(\mathbf{u}) \ln ^{+} f(\mathbf{u})$ is integrable, then

$$
\lim _{n_{1} \geq n_{2} \rightarrow \infty} \frac{1}{n_{1} n_{2}} E\left|S_{n_{1}, n_{2}}(\mathbf{t})\right|^{2}=(2 \pi)^{2} f(\mathbf{t}) .
$$

\section{Stationary random fields and stationary filtrations}

In order to construct stationary filtrations, we shall start with a strictly stationary real valued random field $\xi=\left(\xi_{\mathbf{u}}\right)_{\mathbf{u} \in Z^{2}}$, defined on a probability space $(\Omega, \mathcal{K}, P)$ and define the filtrations

$$
\mathcal{F}_{k, \ell}=\sigma\left(\xi_{j, u}: j \leq k, u \leq \ell\right)
$$

To ease the notation, sometimes the conditional expectation will be denoted by

$$
E_{a, b} X=E\left(X \mid \mathcal{F}_{a, b}\right) .
$$

We shall consider that the filtration is commuting in the sense that

$$
E_{u, v} E_{a, b} X=E_{a \wedge u, b \wedge v} X .
$$

It is remarkable that, by Problem 34.11 in Billingsley [2] (see Lemma 12), condition (6) is equivalent to the apparently weaker condition: for $a \geq u$ and $X$ integrable we have

$$
E_{u, v} E_{a, b} X=E_{u, b \wedge v} X
$$

Now we introduce the stationary random field $\left(X_{\mathbf{m}}\right)_{\mathbf{m} \in Z^{2}}$, in the following way. We define first

$$
X_{\mathbf{0}}=g\left(\left(\xi_{\mathbf{u}}\right)_{\mathbf{u} \in Z^{2}}\right)
$$

where $g: R^{Z^{2}} \rightarrow \mathbb{C}$ and $\mathbf{0}=(0,0)$.

Without restricting the generality, we shall define $\left(\xi_{\mathbf{u}}\right)_{\mathbf{u} \in Z^{2}}$ in a canonical way on the probability space $\Omega=R^{Z^{2}}$, endowed with the $\sigma$-field, $\mathcal{B}(\Omega)$, generated by cylinders. Then, if $\omega=\left(x_{\mathbf{v}}\right)_{\mathbf{v} \in Z^{2}}$ we define $\xi_{\mathbf{u}}^{\prime}(\omega)=x_{\mathbf{u}}$. We construct a probability measure $P^{\prime}$ on $\mathcal{B}(\Omega)$ such that for all $B \in \mathcal{B}(\Omega)$ and any $m$ and $\mathbf{u}_{1}, \ldots, \mathbf{u}_{m}$ we have

$$
P^{\prime}\left(\left(x_{\mathbf{u}_{1}}, \ldots, x_{\mathbf{u}_{m}}\right) \in B\right)=P\left(\left(\xi_{\mathbf{u}_{1}}, \ldots, \xi_{\mathbf{u}_{m}}\right) \in B\right) .
$$

The new sequence $\left(\xi_{\mathbf{u}}^{\prime}\right)_{\mathbf{u} \in Z^{2}}$ is distributed as $\left(\xi_{\mathbf{u}}\right)_{\mathbf{u} \in Z^{2}}$ and re-denoted $\left(\xi_{\mathbf{u}}\right)_{\mathbf{u} \in Z^{2}}$. We shall also redenote $P^{\prime}$ as $P$. Now on $R^{Z^{2}}$ we introduce the operators

$$
T^{\mathbf{u}}\left(\left(x_{\mathbf{v}}\right)_{\mathbf{v} \in Z^{2}}\right)=\left(x_{\mathbf{v}+\mathbf{u}}\right)_{\mathbf{v} \in Z^{2}} .
$$

Two of them will play an important role in our paper namely, when $\mathbf{u}=(1,0)$ and when $\mathbf{u}=$ $(0,1)$. By interpreting the indexes as notations for the lines and columns of a matrix, we shall call

$$
T\left(\left(x_{u, v}\right)_{(u, v) \in Z^{2}}\right)=\left(x_{u+1, v}\right)_{(u, v) \in Z^{2}}
$$


the vertical shift and

$$
S\left(\left(x_{u, v}\right)_{(u, v) \in Z^{2}}\right)=\left(x_{u, v+1}\right)_{(u, v) \in Z^{2}}
$$

the horizontal shift. Then define

$$
X_{j, k}=g\left(T^{j} S^{k}\left(\xi_{\mathbf{u}}\right)_{\mathbf{u} \in Z^{2}}\right)
$$

\section{Results and proofs}

\section{Spectral density representation in terms of projections}

In this section, we first find a useful representation of the spectral density for regular functions and commuting filtrations. It extends a result of Lifshitz and Peligrad [21] beyond the case of Bernoulli shifts. The proof follows the same lines as in Lifshitz and Peligrad [21]. We shall point out the differences and give it here for completeness, clarification and equivalent definitions.

For an integrable random variable $X$, we introduce the projection operators by defining

$$
\begin{aligned}
& P_{\tilde{0}, 0} X=\left(E_{0,0}-E_{-1,0}\right) X, \\
& P_{0, \tilde{0}} X=\left(E_{0,0}-E_{0,-1}\right) X .
\end{aligned}
$$

Note that, by (6), we have

$$
\mathcal{P}_{\mathbf{0}} X=P_{\tilde{0}, 0} P_{0, \tilde{0}} X=P_{0, \tilde{0}} P_{\tilde{0}, 0} X
$$

and by stationarity, for all $u, v \in Z$

$$
\mathcal{P}_{u, v} X=E_{u, v} X-E_{u, v-1} X-E_{u-1, v} X+E_{u-1, v-1} X .
$$

Define $\mathcal{F}_{-\infty, m}=\bigcap_{u \in Z} \mathcal{F}_{u, m}$ and $\mathcal{F}_{m,-\infty}=\bigcap_{v \in Z} \mathcal{F}_{m, v}$.

Now let $X_{\mathbf{0}}$ be defined as before, in $L_{2}(\Omega, \mathcal{F}, P)$. Then we have the following orthogonal representation

$$
X_{\mathbf{0}}=\sum_{\mathbf{u} \in \mathbb{J}_{n, m}} \mathcal{P}_{\mathbf{u}} X_{\mathbf{0}}+R_{n m}+U_{n m},
$$

where $n$ and $m$ are two positive integers, $\mathbb{J}_{n, m}=[-n, \ldots, n] \times[-m, \ldots, m]$,

$$
R_{n m}=E\left(X_{\mathbf{0}} \mid \mathcal{F}_{-n-1, m}\right)+E\left(X_{\mathbf{0}} \mid \mathcal{F}_{n,-m-1}\right)-E\left(X_{\mathbf{0}} \mid \mathcal{F}_{-n-1,-m-1}\right),
$$

and

$$
U_{n m}=X_{\mathbf{0}}-E\left(X_{\mathbf{0}} \mid \mathcal{F}_{n, m}\right) .
$$

We assume the following two regularity conditions

$$
E\left(X_{\mathbf{0}} \mid \mathcal{F}_{-\infty, 0}\right)=0 \quad \text { a.s. and } E\left(X_{\mathbf{0}} \mid \mathcal{F}_{-\infty, 0}\right) \quad \text { a.s. }
$$


Note that

$$
E\left(X_{\mathbf{0}} \mid \mathcal{F}_{-n-1,-m-1}\right)=E\left(E\left(X_{\mathbf{0}} \mid \mathcal{F}_{0,-m-1}\right) \mid \mathcal{F}_{-n-1,0}\right) .
$$

By passing to the limit and using the reverse martingale theorem and arguments similar to Theorem 34.2(V) in Billingsley [2], we obtain that

$$
\lim _{n \rightarrow \infty} \lim _{m \rightarrow \infty} R_{n m}=0 \quad \text { a.s. and in } L_{2} \text {. }
$$

Since $X_{\mathbf{0}}$ is measurable with respect to $\bigvee_{\mathbf{u} \in Z^{2}} \mathcal{F}_{\mathbf{u}}=\mathcal{F}_{\infty, \infty}$, by the martingale convergence theorem,

$$
\lim _{n \rightarrow \infty} \lim _{m \rightarrow \infty} U_{n m}=0 \quad \text { a.s. and in } L_{2} \text {. }
$$

Therefore,

$$
X_{\mathbf{0}}=\lim _{n \rightarrow \infty} \lim _{m \rightarrow \infty} \sum_{j=-n}^{n} \sum_{k=-m}^{m} \mathcal{P}_{-j,-k} X_{\mathbf{0}} \quad \text { a.s. and in } L_{2} .
$$

We shall denote this limit by

$$
X_{\mathbf{0}}=\sum_{\mathbf{u} \in Z^{2}} \mathcal{P}_{\mathbf{u}} X_{\mathbf{0}}
$$

Note that for $\mathbf{u} \neq \mathbf{v}$ and for all $X$ and $Y$ in $L_{2}(\Omega, \mathcal{K}, P)$ we have

$$
\operatorname{cov}\left(\mathcal{P}_{\mathbf{u}} X, \mathcal{P}_{\mathbf{v}} Y\right)=0
$$

Observe also that, by taking into account (11), (12) and stationarity, we have

$$
\sum_{\mathbf{u} \in Z^{2}} E\left|\mathcal{P}_{\mathbf{u}} X_{\mathbf{0}}\right|^{2}=E\left|X_{\mathbf{0}}\right|^{2}<\infty
$$

We would like now to define a random variable which will be used to characterize the spectral density of random fields. For random variables, this was achieved in Peligrad and Wu [24] by using Carleson [4] and also Hunt and Young [18] theorems. For random fields, these theorems do not hold in general. We could use instead a weaker form of them or, as an alternative, an iterated procedure.

In the sequel, we shall use the notation $\|X\|^{2}=E\left(|X|^{2}\right)$.

\section{Martingale difference construction}

We start from the identity (13) and note that this identity implies

$$
\sum_{\mathbf{u} \in Z^{2}}\left|\mathcal{P}_{\mathbf{u}} X_{\mathbf{0}}\right|^{2}<\infty \quad P \text {-a.s. }
$$


Let $\Omega^{\prime} \subset \Omega$ with $P\left(\Omega^{\prime}\right)=1$ be such that the convergence above holds for all $\omega \in \Omega^{\prime}$. By the main theorem in Fefferman [9] for convergence of double Fourier series, we obtain the almost sure convergence in the following sense: For $\omega \in \Omega^{\prime}$ we have

$$
\sum_{\mathbf{j} \in Z^{2}} \mathrm{e}^{-\mathrm{i} \mathbf{j} \cdot \mathbf{t}} \mathcal{P}_{\mathbf{0}} X_{\mathbf{j}}(\omega)=\lim _{n \rightarrow \infty} \sum_{\mathbf{j} \in \mathbb{I}_{n}} \mathrm{e}^{-\mathrm{i} \mathbf{j} \cdot \mathbf{t}} \mathcal{P}_{\mathbf{0}} X_{\mathbf{j}}(\omega) \quad \lambda^{2} \text {-a.e. }
$$

where $\mathbb{I}_{n}=[-n, n] \times[-n, n]$.

By Fubini theorem, for almost all $\mathbf{t} \in I$, we also have that

$$
\sum_{\mathbf{j} \in Z^{2}} \mathrm{e}^{-\mathrm{ij} \cdot \mathbf{t}} \mathcal{P}_{\mathbf{0}} X_{\mathbf{j}}=\lim _{n \rightarrow \infty} \sum_{\mathbf{j} \in \mathbb{I}_{n}} \mathrm{e}^{-\mathrm{ij} \cdot \mathbf{t}} \mathcal{P}_{\mathbf{0}} X_{\mathbf{j}} \quad P \text {-a.s. }
$$

Furthermore, by relation (1) in Fefferman's paper [9] and by (14), for a positive constant $C$, we have that

$$
\int_{I} \sup _{n}\left|\sum_{\mathbf{j} \in \mathbb{I}_{n}} \mathrm{e}^{-\mathrm{ij} \cdot \mathbf{t}} \mathcal{P}_{\mathbf{0}} X_{\mathbf{j}}\right|^{2} \mathrm{~d} \mathbf{t} \leq C \sum_{\mathbf{u} \in Z^{2}}\left|\mathcal{P}_{\mathbf{u}} X_{\mathbf{0}}\right|^{2} \quad P \text {-a.s. }
$$

Whence, by integrating and using (13), we obtain

$$
E \int_{I} \sup _{n}\left|\sum_{\mathbf{j} \in \mathbb{I}_{n}} \mathrm{e}^{-\mathrm{i} \mathbf{j} \cdot \mathbf{t}} \mathcal{P}_{\mathbf{0}} X_{\mathbf{j}}\right|^{2} \mathrm{~d} \mathbf{t} \leq C E \sum_{\mathbf{u} \in Z^{2}}\left|\mathcal{P}_{\mathbf{u}} X_{\mathbf{0}}\right|^{2} \leq C\left\|X_{\mathbf{0}}\right\|^{2} .
$$

It follows that for almost all $\mathbf{t} \in I$

$$
E\left(\sup _{n}\left|\sum_{\mathbf{j} \in \mathbb{I}_{n}} \mathrm{e}^{-\mathrm{ij} \cdot \mathbf{t}} \mathcal{P}_{\mathbf{0}} X_{\mathbf{j}}\right|^{2}\right)<\infty
$$

By the dominated convergence theorem, the convergence in (15) also holds in $L_{2}$.

Let us denote by

$$
D_{\mathbf{0}}(\mathbf{t})=\lim _{n \rightarrow \infty} \sum_{\mathbf{j} \in \mathbb{I}_{n}} \mathrm{e}^{-\mathrm{ij} \cdot \mathbf{t}} \mathcal{P}_{\mathbf{0}} X_{\mathbf{j}} \quad P \text {-a.s. and in } L_{2} .
$$

In the next theorem, we point out a representations for the spectral density by using definition (16).

Theorem 1. Let $\left(X_{\mathbf{k}}\right)_{\mathbf{k} \in Z^{2}}$ be a stationary sequence defined by (8) and the filtration $\left(\mathcal{F}_{\mathbf{k}}\right)_{\mathbf{k} \in Z^{2}}$ is commuting as in (6). Assume that the second moment is finite and the regularity condition (10) is satisfied. Then, the sequence $\left(X_{\mathbf{k}}\right)_{\mathbf{k} \in Z^{2}}$ has spectral density which has the representation:

$$
f(\mathbf{t})=\frac{1}{(2 \pi)^{2}} E\left|D_{\mathbf{0}}(\mathbf{t})\right|^{2}, \quad \mathbf{t} \in I .
$$


Proof of Theorem 1. Let us compute the covariance of $X_{\mathbf{k}}$ and $X_{\mathbf{0}}$. By using the projection decomposition in (11), written for both $X_{\mathbf{k}}$ and $X_{\mathbf{0}}$, together with the orthogonality of the projections in (12) and stationarity, we have for all $\mathbf{k} \in Z^{2}$,

$$
\begin{aligned}
\operatorname{cov}\left(X_{\mathbf{k}}, X_{\mathbf{0}}\right) & =\operatorname{cov}\left(\sum_{\mathbf{j} \in Z^{2}} \mathcal{P}_{\mathbf{j}} X_{\mathbf{k}}, \sum_{\mathbf{u} \in Z^{2}} \mathcal{P}_{\mathbf{u}} X_{\mathbf{0}}\right) \\
& =\sum_{\mathbf{j} \in Z^{2}} \operatorname{cov}\left(\mathcal{P}_{\mathbf{j}} X_{\mathbf{k}}, \mathcal{P}_{\mathbf{j}} X_{\mathbf{0}}\right)=\sum_{\mathbf{j} \in Z^{2}} \operatorname{cov}\left(\mathcal{P}_{\mathbf{0}} X_{\mathbf{k}+\mathbf{j}}, \mathcal{P}_{\mathbf{0}} X_{\mathbf{j}}\right)
\end{aligned}
$$

Let us analyze the function $f(\mathbf{t})$ defined in (17). By Fubini theorem and (13) we have

$$
\int_{I} f(\mathbf{t}) \mathrm{d} \mathbf{t}=\frac{1}{(2 \pi)^{2}} E \int_{I}\left|\sum_{\mathbf{j} \in Z^{2}} \mathrm{e}^{-\mathrm{ij} \cdot \mathbf{t}} \mathcal{P}_{\mathbf{0}} X_{\mathbf{j}}\right|^{2} \mathrm{~d} \mathbf{t}=E \sum_{\mathbf{j} \in Z^{2}}\left|\mathcal{P}_{\mathbf{j}} X_{\mathbf{0}}\right|^{2}<\infty .
$$

Now, let us compute the Fourier coefficients of $f(\mathbf{t})$. For every $\mathbf{k} \in Z^{2}$, by the definition of $f(\mathbf{t})$ and Fubini theorem we have

$$
\int_{I} \mathrm{e}^{\mathrm{i} \mathbf{k} \cdot \mathbf{t}} f(\mathbf{t}) \mathrm{d} \mathbf{t}=\frac{1}{(2 \pi)^{2}} \sum_{\mathbf{u}, \mathbf{j} \in Z^{2}} E \int_{I} \mathrm{e}^{\mathrm{i}(\mathbf{k}-\mathbf{j}+\mathbf{u}) \cdot \mathbf{t}} \mathcal{P}_{\mathbf{0}} X_{\mathbf{j}} \overline{\mathcal{P}_{\mathbf{0}} X_{\mathbf{u}}} \mathrm{d} \mathbf{t} .
$$

By using the orthogonality of the exponential functions, we obtain

$$
\begin{aligned}
\int_{I} \mathrm{e}^{\mathrm{i} \mathbf{k} \cdot \mathbf{t}} f(\mathbf{t}) \mathrm{d} \mathbf{t} & =\sum_{\mathbf{j}, \mathbf{u} \in Z^{2}} \operatorname{cov}\left(\mathcal{P}_{\mathbf{0}} X_{\mathbf{j}}, \mathcal{P}_{\mathbf{0}} X_{\mathbf{u}}\right) \mathbf{1}_{\{\mathbf{k}-\mathbf{j}+\mathbf{u}=\mathbf{0}\}} \\
& =\sum_{\mathbf{u} \in Z^{2}} \operatorname{cov}\left(\mathcal{P}_{\mathbf{0}} X_{\mathbf{u}+\mathbf{k}}, \mathcal{P}_{\mathbf{0}} X_{\mathbf{u}}\right)
\end{aligned}
$$

Now, comparing this expression with (18) we see that $f$ in formula (17) is the spectral density for $\left(X_{\mathbf{k}}\right)_{\mathbf{k} \in Z^{2}}$.

Remark 2. For defining the spectral density iterated limits are also possible. By applying Carleson [4] and Hunt and Young [18] theorems twice, consecutively in each variable, one can show that the following limits exist: for $\lambda^{2}$-almost all $\mathbf{t} \in[-\pi, \pi)^{2}$, we can define a random variable $\tilde{D}_{\mathbf{0}}(\mathbf{t})$ in the following sense

$$
\tilde{D}_{\mathbf{0}}(\mathbf{t})=\lim _{n \rightarrow \infty} \lim _{m \rightarrow \infty} \sum_{u_{1}=-n}^{n} \sum_{u_{2}=-m}^{m} \mathcal{P}_{\mathbf{0}}\left(X_{u_{1}, u_{2}}\right) \mathrm{e}^{- \text {iu.t }} \quad P \text {-a.s. and in } L_{2} .
$$

Similarly, we can also define the other iterated limit

$$
\hat{D}_{\mathbf{0}}(\mathbf{t})=\lim _{m \rightarrow \infty} \lim _{n \rightarrow \infty} \sum_{u_{1}=-n}^{n} \sum_{u_{2}=-m}^{m} \mathcal{P}_{\mathbf{0}}\left(X_{u_{1}, u_{2}}\right) \mathrm{e}^{-\mathrm{iu} \cdot \mathbf{t}} \quad P \text {-a.s. and in } L_{2} .
$$


Also, we can obtain the following alternative definitions for the spectral density:

$$
f(\mathbf{t})=\frac{1}{(2 \pi)^{2}} E\left|\hat{D}_{\mathbf{0}}(\mathbf{t})\right|^{2}=\frac{1}{(2 \pi)^{2}} E\left|\tilde{D}_{\mathbf{0}}(\mathbf{t})\right|^{2} .
$$

Note that in all the characterizations of $f(\mathbf{t})$ the limits commute with the integrals.

Remark 3. By (6) and its definition, $D_{\mathbf{0}}(\mathbf{t})$ is a martingale difference in each coordinate

$$
E_{0,-1} D_{\mathbf{0}}(\mathbf{t})=0 \quad \text { and } \quad E_{-1,0} D_{\mathbf{0}}(\mathbf{t})=0 \quad P \text {-a.s. }
$$

We are ready to state our main result. Everywhere in the paper $\Rightarrow$ denotes convergence in distribution.

Theorem 4. Assume that $\left(X_{\mathbf{k}}\right)_{\mathbf{k} \in Z^{2}}$ and $\left(\mathcal{F}_{\mathbf{k}}\right)_{\mathbf{k} \in Z^{2}}$ are as in Theorem 1. In addition, assume that one of the shifts $T$ or $S$ is ergodic. Then, for $\lambda^{2}$-almost all $\mathbf{t} \in I$,

$$
\frac{1}{n}\left(\operatorname{Re} S_{n, n}(\mathbf{t}), \operatorname{Im} S_{n, n}(\mathbf{t})\right) \Rightarrow\left(N_{1}, N_{2}\right) \quad \text { as } n \rightarrow \infty,
$$

where $N_{1}, N_{2}$ are i.i.d. normally distributed random variables with mean 0 and variance $2 \pi^{2} f(\mathbf{t})$, where $f(\mathbf{t})$ is the spectral density of the sequence $\left(X_{\mathbf{k}}\right)_{\mathbf{k} \in Z^{2}}$. Furthermore, if $f(\mathbf{u}) \ln ^{+} f(\mathbf{u})$ is integrable then

$$
\frac{1}{\sqrt{n_{1} n_{2}}}\left(\operatorname{Re} S_{n_{1}, n_{2}}(\mathbf{t}), \operatorname{Im} S_{n_{1}, n_{2}}(\mathbf{t})\right) \Rightarrow\left(N_{1}, N_{2}\right) \quad \text { as } n_{1} \wedge n_{2} \rightarrow \infty
$$

with $N_{1}, N_{2}$ as above.

Proof of Theorem 4. This proof has several steps. Let us point out the idea of the proof. First we show that the proof can be reduced to random variables with continuous spectral density. Then, we construct a random field which is a martingale difference in each coordinate and has the same limiting distribution as the original sigma field. To validate this approximation we shall use the limiting variance given in (3) and (4) along with the representation of the spectral density given in Theorem 1. The result will follow by obtaining the central limit theorem for the martingale random field. To fix the ideas let us assume that the shift $S$ is ergodic.

\section{Martingale approximation}

Let us recall the definition of $D_{\mathbf{0}}(\mathbf{t})$ given in (16) and introduce a new notation:

$$
D_{\mathbf{0}}^{(\ell)}(\mathbf{t})=\sum_{\mathbf{j} \in \mathbb{I}_{\ell}} \mathcal{P}_{\mathbf{0}}\left(X_{\mathbf{j}}\right) \mathrm{e}^{-\mathrm{i} \mathbf{j} \cdot \mathbf{t}} \rightarrow \sum_{\mathbf{j} \in Z^{2}} \mathcal{P}_{\mathbf{0}}\left(X_{\mathbf{j}}\right) \mathrm{e}^{-\mathrm{ij} \cdot \mathbf{t}}=D_{\mathbf{0}}(\mathbf{t}) \quad P \text {-a.s. and in } L_{2} .
$$

Note that $D_{\mathbf{0}}(\mathbf{t})$ and $D_{\mathbf{0}}^{(\ell)}(\mathbf{t})$ are functions of $\left(\boldsymbol{\xi}_{\mathbf{u}}\right)_{\mathbf{u} \in Z^{2}}$. By using stationarity and translation operators $T$ and $S$ we define $D_{\mathbf{k}}^{(\ell)}(\mathbf{t})$ and $D_{\mathbf{k}}(\mathbf{t})$ for any $\mathbf{k} \in Z^{2}$. Note that, by Remark 3 , both 
$\left(D_{u, v}^{(\ell)}(\mathbf{t})\right)$ and $\left(D_{u, v}(\mathbf{t})\right)$ are coordinate-wise martingale differences with respect to the filtrations $\left(\mathcal{F}_{\infty, v}\right)_{v}$ and $\left(\mathcal{F}_{u, \infty}\right)_{u}$ respectively.

For almost all $\mathbf{t} \in I$ we shall approximate $S_{\mathbf{n}}(\mathbf{t})$ by the martingale

$$
M_{\mathbf{n}}(\mathbf{t})=\sum_{\mathbf{j}=\mathbf{1}}^{\mathbf{n}} \mathrm{e}^{\mathrm{i} \mathbf{j} \cdot \mathbf{t}} D_{\mathbf{j}}(\mathbf{t})
$$

To validate this approximation, we first consider the situation when $\mathbf{n}=(n, n)$. Define the martingale

$$
M_{\mathbf{n}}^{(\ell)}(\mathbf{t})=\sum_{\mathbf{j}=\mathbf{1}}^{\mathbf{n}} \mathrm{e}^{\mathrm{ij} \cdot \mathbf{t}} D_{\mathbf{j}}^{(\ell)}(\mathbf{t})
$$

and, for $\mathbf{t}^{\prime}$ fixed, the "proper" Fourier series in $\mathbf{t}$,

$$
M_{\mathbf{n}}^{(\ell)}\left(\mathbf{t}, \mathbf{t}^{\prime}\right)=\sum_{\mathbf{j}=\mathbf{1}}^{\mathbf{n}} \mathrm{e}^{\mathrm{i} \mathbf{j} \cdot \mathbf{t}} D_{\mathbf{j}}^{(\ell)}\left(\mathbf{t}^{\prime}\right)
$$

Note that we can bound

$$
\begin{aligned}
& \left|S_{\mathbf{n}}(\mathbf{t})-M_{\mathbf{n}}(\mathbf{t})\right|^{2} \\
& \quad \leq 3\left(\left|S_{\mathbf{n}}(\mathbf{t})-M_{\mathbf{n}}^{(\ell)}\left(\mathbf{t}, \mathbf{t}^{\prime}\right)\right|^{2}+\left|M_{\mathbf{n}}^{(\ell)}\left(\mathbf{t}, \mathbf{t}^{\prime}\right)-M_{\mathbf{n}}^{(\ell)}(\mathbf{t})\right|^{2}+\left|M_{\mathbf{n}}^{(\ell)}(\mathbf{t})-M_{\mathbf{n}}(\mathbf{t})\right|^{2}\right) .
\end{aligned}
$$

By (3), for almost all $\mathbf{t} \in I$

$$
\lim _{n \rightarrow \infty} \frac{1}{n^{2}} E\left|S_{\mathbf{n}}(\mathbf{t})-M_{\mathbf{n}}^{(\ell)}\left(\mathbf{t}, \mathbf{t}^{\prime}\right)\right|^{2}=(2 \pi)^{2} f^{(\ell)}\left(\mathbf{t}, \mathbf{t}^{\prime}\right)
$$

where $f^{(\ell)}\left(\mathbf{t}, \mathbf{t}^{\prime}\right)$ is the spectral density of $\left(X_{\mathbf{k}}-D_{\mathbf{k}}^{(\ell)}\left(\mathbf{t}^{\prime}\right)\right)_{\mathbf{k}}$.

By using the representation (17) given in Theorem 1, and taking into account that $\mathcal{P}_{\mathbf{0}} D_{\mathbf{j}}^{(\ell)}\left(\mathbf{t}^{\prime}\right)=$ $0 P$-a.s. for $\mathbf{j} \in Z^{2}$ with $\mathbf{j} \neq \mathbf{0}$ we obtain

$$
f^{(\ell)}\left(\mathbf{t}, \mathbf{t}^{\prime}\right)=\frac{1}{(2 \pi)^{2}} E\left|\sum_{\mathbf{j} \in Z_{2}} \mathcal{P}_{\mathbf{0}} X_{\mathbf{j}} \mathrm{e}^{-\mathrm{ij} \cdot \mathbf{t}}-D_{\mathbf{0}}^{(\ell)}\left(\mathbf{t}^{\prime}\right)\right|^{2}=\frac{1}{(2 \pi)^{2}} E\left|D_{\mathbf{0}}(\mathbf{t})-D_{\mathbf{0}}^{(\ell)}\left(\mathbf{t}^{\prime}\right)\right|^{2} .
$$

On the other hand, by the orthogonality of the projections,

$$
\frac{1}{n^{2}} E\left|M_{\mathbf{n}}^{(\ell)}\left(\mathbf{t}, \mathbf{t}^{\prime}\right)-M_{\mathbf{n}}^{(\ell)}(\mathbf{t})\right|^{2}=E\left|D_{\mathbf{0}}^{(\ell)}\left(\mathbf{t}^{\prime}\right)-D_{\mathbf{0}}^{(\ell)}(\mathbf{t})\right|^{2}
$$

and

$$
\frac{1}{n^{2}} E\left|M_{\mathbf{n}}^{(\ell)}(\mathbf{t})-M_{\mathbf{n}}(\mathbf{t})\right|^{2}=E\left|D_{\mathbf{0}}^{(\ell)}(\mathbf{t})-D_{\mathbf{0}}(\mathbf{t})\right|^{2}
$$


So, overall, by the above considerations,

$$
\begin{aligned}
& \lim _{n \rightarrow \infty} \frac{1}{n^{2}} E\left|S_{\mathbf{n}}(\mathbf{t})-M_{\mathbf{n}}(\mathbf{t})\right|^{2} \\
& \quad \leq 3\left(E\left|D_{0}(\mathbf{t})-D_{\mathbf{0}}^{(\ell)}\left(\mathbf{t}^{\prime}\right)\right|^{2}+E\left|D_{\mathbf{0}}^{(\ell)}\left(\mathbf{t}^{\prime}\right)-D_{\mathbf{0}}^{(\ell)}(\mathbf{t})\right|^{2}+E\left|D_{\mathbf{0}}^{(\ell)}(\mathbf{t})-D_{\mathbf{0}}(\mathbf{t})\right|^{2}\right) .
\end{aligned}
$$

Note now that $D_{\mathbf{0}}^{(\ell)}\left(\mathbf{t}^{\prime}\right)$ is continuous in $\mathbf{t}^{\prime}$ and so, by the dominated convergence theorem, $\lim _{\mathbf{t}^{\prime} \rightarrow \mathbf{t}} D_{\mathbf{0}}^{(\ell)}\left(\mathbf{t}^{\prime}\right)=D_{\mathbf{0}}^{(\ell)}(\mathbf{t})$ in $L_{2}$. Therefore, by taking into account (19), and letting first $\mathbf{t}^{\prime} \rightarrow \mathbf{t}$ and then $\ell \rightarrow \infty$, we obtain for $\lambda^{2}$-almost all $\mathbf{t} \in I$ the approximation

$$
\lim _{n \rightarrow \infty} \frac{1}{n^{2}} E\left|S_{\mathbf{n}}(\mathbf{t})-M_{\mathbf{n}}(\mathbf{t})\right|^{2}=0 .
$$

Furthermore, if $\mathbf{n}=\left(n_{1}, n_{2}\right)$ and $f(\mathbf{u}) \ln ^{+} f(\mathbf{u})$ is integrable, by replacing in the proof the limit given in (3) by (4), for $\lambda^{2}$-almost all $\mathbf{t} \in I$ we have

$$
\lim _{n_{1}>n_{2} \rightarrow \infty} \frac{1}{n_{1} n_{2}} E\left|S_{\mathbf{n}}(\mathbf{t})-M_{\mathbf{n}}(\mathbf{t})\right|^{2}=0 .
$$

By using Theorem 25.4 in Billingsley [2], the limit (23) shows that, the proof of Theorem 4 is now reduced to prove the central limit theorem for $M_{\mathbf{n}}(\mathbf{t})$.

\section{The central limit theorem for the martingale}

Proposition 5. Consider $M_{\mathbf{n}}(\mathbf{t})$ defined by (20) where $\mathbf{n}=\left(n_{1}, n_{2}\right)$. Then the real and imaginary part of $M_{\mathbf{n}}(\mathbf{t})$ converge to independent normal random variables with variance $E\left|D_{0,0}(\mathbf{t})\right|^{2} / 2$ when $n_{1} \wedge n_{2} \rightarrow \infty$.

Proof. To ease the notation, we shall drop $\mathbf{t}$ and denote $D_{j, k}=D_{j, k}(\mathbf{t}), M_{\mathbf{n}}=M_{\mathbf{n}}(\mathbf{t})$.

We start by writing $\left(\mathbf{t}=\left(t_{1}, t_{2}\right)\right)$

$$
\frac{1}{\sqrt{n_{1} n_{2}}} M_{\mathbf{n}}=\frac{1}{\sqrt{n_{1}}} \sum_{j=1}^{n_{1}} e^{\mathrm{i} j t_{1}} \frac{1}{\sqrt{n_{2}}} \sum_{k=1}^{n_{2}} e^{\mathrm{i} k t_{2}} D_{j, k} .
$$

Note that, by construction and since the filtration $\left(\mathcal{F}_{j, k}\right)$ is commuting, the sequence $\left(D_{n_{2}, j}^{\prime}\right)_{j}$ defined by

$$
D_{n_{2}, j}^{\prime}=\frac{1}{\sqrt{n_{2}}} \sum_{k=1}^{n_{2}} e^{\mathrm{i} k t_{2}} D_{j, k}
$$

is a triangular array of complex martingale differences with respect to the filtration $\left(\mathcal{F}_{j, \infty}\right)_{j}$. 
For $a$ and $b$ real numbers let us find the limiting distribution of

$$
\begin{aligned}
a & \frac{1}{\sqrt{n_{1} n_{2}}} \operatorname{Re} M_{\mathbf{n}}+b \frac{1}{\sqrt{n_{1} n_{2}}} \operatorname{Im} M_{\mathbf{n}} \\
& =\frac{1}{\sqrt{n_{1}}} \sum_{j=1}^{n_{1}}\left[\left(a \cos j t_{1}+b \sin j t_{1}\right) \operatorname{Re} D_{n_{2}, j}^{\prime}+\left(b \cos j t_{1}-a \sin j t_{1}\right) \operatorname{Im} D_{n_{2}, j}^{\prime}\right] \\
& =\frac{1}{\sqrt{n_{1}}} \sum_{j=1}^{n_{1}} \Delta_{n_{2}, j} .
\end{aligned}
$$

In order to find the limiting distribution of $n_{1}^{-1 / 2} \sum_{j=1}^{n_{1}} \Delta_{n_{2}, j}$ we have to prove now a central limit theorem for the triangular array of martingale differences $\left(\Delta_{n_{2}, j}\right)_{j \geq 1}$. According to a classical result, which can be found in Gänssler and Häusler [12], we have to establish that

$$
\max _{1 \leq j \leq n_{1}} \frac{1}{\sqrt{n_{1}}}\left|\Delta_{n_{2}, j}\right| \rightarrow^{L_{2}} 0 \quad \text { as } n_{1} \wedge n_{2} \rightarrow \infty .
$$

and to verify the Raikov type condition, namely

$$
\frac{1}{n_{1}} E\left|\sum_{j=1}^{n_{1}}\left(\Delta_{n_{2}, j}^{2}-E \Delta_{n_{2}, j}^{2}\right)\right| \rightarrow 0 \quad \text { as } n_{1} \wedge n_{2} \rightarrow \infty .
$$

The first condition is easy to verify since, by the stationarity involved in the model and the main result in Peligrad and $\mathrm{Wu}[24]$, the variables $\left(\left|D_{n_{2}, j}^{\prime}\right|^{2}\right)_{j}$ are uniformly integrable and therefore, $\left(\left|\Delta_{n_{2}, j}\right|^{2}\right)_{j}$ in (24) are also uniformly integrable.

In order to verify (25), after using the well-known trigonometric formulas

$$
\begin{aligned}
2 \cos ^{2} x & =1+\cos 2 x, \quad 2 \sin ^{2} x=1-\cos 2 x, \\
\cos ^{2} x-\sin ^{2} x & =\cos 2 x, \quad 2(\cos x)(\sin x)=\sin 2 x,
\end{aligned}
$$

by Lemma 10 in Section 6, it follows that for almost all $\mathbf{t} \in I$, the terms involving $\cos 2 j t_{1}$ or $\sin 2 j t_{1}$ in (25) are negligible as $n_{1} \wedge n_{2} \rightarrow \infty$.

After simple computations, proving (25) is reduced to show that

$$
\frac{1}{n_{1}} E\left|\sum_{j=1}^{n_{1}}\left(\left|D_{n_{2}, j}^{\prime}\right|^{2}-E\left|D_{n_{2}, j}^{\prime}\right|^{2}\right)\right| \rightarrow 0
$$

We shall apply Lemma 11 below. Clearly it is enough to prove that

$$
\frac{1}{n_{1}} E\left|\sum_{j=1}^{n_{1}}\left(\operatorname{Re}^{2} D_{n_{2}, j}^{\prime}-E \operatorname{Re}^{2} D_{n_{2}, j}^{\prime}\right)\right| \rightarrow 0
$$


and

$$
\frac{1}{n_{1}} E\left|\sum_{j=1}^{n_{1}}\left(\operatorname{Im}^{2} D_{n_{2}, j}^{\prime}-E \operatorname{Im}^{2} D_{n_{2}, j}^{\prime}\right)\right| \rightarrow 0 .
$$

Their proofs are similar and we shall deal only with the first one involving the real part.

Let $m$ be a positive integer. By using Cramèr theorem, trigonometric formulas, the main Theorem in Peligrad and Wu [24], ergodicity of $S$ and Lemma 10 from Section 6, we can easily show that the vector valued sequence of martingales $\left(\operatorname{Re} D_{n_{2}, 1}^{\prime}, \ldots, \operatorname{Re} D_{n_{2}, m}^{\prime}\right)_{n_{2}}$ converges to a Gaussian vector $\left(N_{1}, \ldots, N_{m}\right)$ with the covariance structure, for all $k$,

$$
\operatorname{cov}\left(N_{1}, N_{j}\right)=\operatorname{cov}\left(N_{k}, N_{k+j}\right) .
$$

The computations are simple and left to the reader. Because of the martingale property, we have the orthogonality of $\left(\operatorname{Re} D_{n_{2}, k}^{\prime}\right)_{k}$. In addition, we also have uniform integrability of the family $\left(\left|D_{n_{2}, 1}^{\prime}\right|^{2}\right)_{n_{2}}$, provided by the results in Peligrad and Wu [24]. By applying the continuous mapping theorem and the convergence of moments theorem associated to the convergence in distribution (Theorem 25.12 in Billingsley [2]), we obtain

$$
\operatorname{cov}\left(N_{1}, N_{k}\right)=\lim _{n_{2} \rightarrow \infty} \operatorname{cov}\left(\operatorname{Re} D_{n_{2}, 1}^{\prime}, \operatorname{Re} D_{n_{2}, k}^{\prime}\right)=0 .
$$

This shows that the Gaussian limit $\left(N_{k}\right)_{k}$ is a stationary and independent sequence. It follows that, for all $m \in N,\left(\operatorname{Re}^{2} D_{n_{2}, 1}^{\prime}, \ldots, \operatorname{Re}^{2} D_{n_{2}, m}^{\prime}\right)_{n_{2}}$ converges to an independent vector $\left(N_{1}^{2}, \ldots, N_{m}^{2}\right)$ and $\left(N_{k}^{2}\right)_{k}$ is stationary and ergodic. Therefore, (25) holds by Lemma 11 in Section 6.

By all of the above considerations, we obtain

$$
a \frac{1}{\sqrt{n_{1} n_{2}}} \operatorname{Re} M_{\mathbf{n}}(\mathbf{t})+b \frac{1}{\sqrt{n_{1} n_{2}}} \operatorname{Im} M_{\mathbf{n}}(\mathbf{t}) \Rightarrow\left(a^{2}+b^{2}\right) N\left(0, E\left|D_{0,0}(\mathbf{t})\right|^{2}\right),
$$

and the result follows.

\section{Random fields with multi-dimensional index set}

In this section, we discuss the differences which occur when the index set is $Z^{d}$. The main difference is that we use some recent results on summability of multi-dimensional trigonometric Fourier series which are surveyed and further developed in Weisz [31]. Many summability results, needed for our proofs, have already been extended from dimension 1 to dimension $d$, but the results are very different depending on the summation type and on the shape of the regions in $Z^{d}$ containing the indexes of summations. Our intention is to present a method rather than the most general results. The probabilistic tools are completely developed in our paper. However, the statements are limited by the level of knowledge in harmonic analysis. In order to construct the approximating martingale we can always base ourselves on the summation on cubes, where 
the celebrated Carleson-Hunt theorem extends completely for square integrable functions (see Theorem 4.4. in Weisz [31]) or we can use an iterative procedure. However, the statements of the CLT and the conditions imposed to the spectral density, strongly depend on shape of the summation region and the extensions of the Fejér-Lebesgue theorem, namely on the validity of (3). These regions of summation can be restricted by using conditions imposed to various norms on $Z^{d}$ or the summations can be taken over nonrestricted rectangles. In the latter case, additional restrictions have to be imposed to the spectral density. This is an active field of research in harmonic analysis and our results can be reformulated whenever a progress is achieved. We shall formulate the general results by using only summations over cubes and nonrestricted rectangles.

To introduce the regularity conditions we shall start with a strictly stationary real valued random field $\xi=\left(\xi_{\mathbf{u}}\right)_{\mathbf{u} \in Z^{d}}$, defined on the canonical probability space $R^{Z^{d}}$ and define the filtrations $\mathcal{F}_{\mathbf{u}}=\sigma\left(\xi_{\mathbf{j}}: \mathbf{j} \leq \mathbf{u}\right)$. Recall that by $\mathbf{j} \leq \mathbf{u}$ we understand that each coordinate of $\mathbf{j}$ is less or equal the corresponding coordinate of $\mathbf{u}$. By taking intersections of sigma algebras or sigma algebra generated by unions of sigma algebras, we can consider the coordinates of $\mathbf{u}$ in $\mathcal{F}_{\mathbf{u}}$ being valued in $Z \cup\{-\infty, \infty\}$. The filtration is commuting if $E_{\mathbf{u}} E_{\mathbf{a}} X=E_{\mathbf{u} \wedge \mathbf{a}} X$, where the minimum is taken coordinate-wise. We define $X_{\mathbf{0}}=f\left(\left(\xi_{\mathbf{u}}\right)_{\mathbf{u} \in Z^{d}}\right)$. We also define $T_{i}$ the coordinate-wise translations and $X_{\mathbf{k}}=f\left(T_{1}^{k_{1}} \circ \cdots \circ T_{d}^{k_{d}}\left(\xi_{\mathbf{u}}\right)_{\mathbf{u} \in Z^{d}}\right)$. We call $f$ regular if $E\left(X_{\mathbf{0}} \mid \mathcal{F}_{\mathbf{u}}\right)=0$ a.s., when at least a coordinate of $\mathbf{u}$ is $-\infty$.

Our general result is summarized in the following theorem.

Theorem 6. Assume that $\left(X_{\mathbf{k}}\right)_{\mathbf{k} \in Z^{d}}$ and $\left(\mathcal{F}_{\mathbf{k}}\right)_{\mathbf{k} \in Z^{d}}$ are as above and $f$ is regular. In addition, assume that one of the shifts $T_{i}$ is ergodic, $1 \leq i \leq d$. Then, for $\lambda^{d}$-almost all $\mathbf{t} \in[0,2 \pi)^{d}$,

$$
\frac{1}{n^{d / 2}}\left(\operatorname{Re} S_{\mathbf{n}}(\mathbf{t}), \operatorname{Im} S_{\mathbf{n}}(\mathbf{t})\right) \Rightarrow\left(N_{1}, N_{2}\right) \quad \text { as } n \rightarrow \infty
$$

where $\mathbf{n}=(n, \ldots, n), N_{1}, N_{2}$ are i.i.d. normally distributed random variables with mean 0 and variance $2^{d-1} \pi^{d} f(\mathbf{t})$, and $f(\mathbf{t})$ is the spectral density of the sequence $\left(X_{\mathbf{k}}\right)_{\mathbf{k} \in Z^{d}}$. Furthermore, if $f(\mathbf{u})\left(\ln ^{+} f(\mathbf{u})\right)^{d-1}$ is integrable, then

$$
\frac{1}{\sqrt{n_{1} n_{2} \cdots n_{d}}}\left(\operatorname{Re} S_{\mathbf{n}}(\mathbf{t}), \operatorname{Im} S_{\mathbf{n}}(\mathbf{t})\right) \Rightarrow\left(N_{1}, N_{2}\right) \quad \text { as } \bigwedge_{1 \leq i \leq d} n_{i} \rightarrow \infty,
$$

with $N_{1}, N_{2}$ as above.

Proof of Theorem 6. The proof of this theorem follows the same lines as of Theorem 4 with the following differences. In order to be able to obtain a characterization of the spectral density, we have to introduce the $d$-dimensional projection operator. By using the commutative property of the filtrations it is convenient to define

$$
\mathcal{P}_{\mathbf{0}}(X)=\mathcal{P}_{1} \circ \mathcal{P}_{2} \circ \cdots \circ \mathcal{P}_{d}(X)
$$

where

$$
\mathcal{P}_{j}(Y)=E\left(Y \mid \mathcal{F}_{0}^{(j)}\right)-E\left(Y \mid \mathcal{F}_{-1}^{(j)}\right)
$$


Above we used the notation: $\mathcal{F}_{0}^{(j)}=\mathcal{F}_{\mathbf{0}}$, and $\mathcal{F}_{-1}^{(j)}=\mathcal{F}_{\mathbf{u}}$, where $\mathbf{u}$ has all the coordinates 0 with the exception of the $j$ th coordinate, which is -1 . For instance when $d=3, \mathcal{P}_{2}(Y)=$ $E\left(Y \mid \mathcal{F}_{0,0,0}\right)-E\left(Y \mid \mathcal{F}_{0,-1,0}\right)$.

We can easily see that, by using the commutativity property, this definition is a generalization of the case $d=2$. We note that, by using this definition of $\mathcal{P}_{\mathbf{0}}(X)$, the statement and the proof of Theorem 1 remain unchanged if we replace $Z^{2}$ with $Z^{d}$. The definition of the approximating martingale is also clear as well as the proof. We point out the following two differences in the proof. One difference is that instead of Theorem 1 in Marcinkiewicz and Zygmund [22] we use Corollary 14.4 in Weisz [31], which assures the validity of (3) for $\lambda^{d}$-almost all $\mathbf{t}$ in $[0,2 \pi)^{d}$. Another difference in the proof is that Proposition 5 is proved by induction. More precisely, we use instead of the results in Peligrad and $\mathrm{Wu}$ [24] the induction hypothesis. For proving the second part of the Theorem 6, we use several results in Weisz [31], namely Corollary 16.5 about unrestricted summability and the line above relation 15.2 on page 123 .

\section{Examples}

We start this section by mentioning an easy way to generate commuting filtrations. This happens for instance, when we consider a stationary random field $\boldsymbol{\xi}=\left(\overline{\boldsymbol{\xi}}_{\ell}\right)_{\ell \in Z}$ with its columns $\overline{\boldsymbol{\xi}}_{\ell}=$ $\left(\xi_{u, \ell}\right)_{u \in Z}$ independent copies of a stationary stochastic process. Of course, we can also consider as well random fields with independent lines. This can be seen by combining Lemmas 13 and 12 in Section 6. The random field of interest is then constructed by taking functions of $\left(\xi_{k, j}\right)_{k, j \in Z}$ as in definition (8). Furthermore, if the columns of $\left(\xi_{k, j}\right)$ are independent, then $\mathcal{F}_{0,-\infty}$ is trivial. If the lines of $\left(\xi_{k, j}\right)_{k, j \in Z}$ are independent then $\mathcal{F}_{-\infty, 0}$ is trivial.

Next, we give examples of stationary random fields $\left(\xi_{\mathbf{u}}\right)_{\mathbf{u} \in Z^{2}}$ which generate commuting filtration and in addition both $\mathcal{F}_{0,-\infty}$ and $\mathcal{F}_{-\infty, 0}$ are trivial.

\section{Independent copies of a stationary sequence with "nonparallel" past and future}

The $\rho$-mixing coefficient, also known as maximal coefficient of correlation is defined as

$$
\rho(\mathcal{A}, \mathcal{B})=\sup \left\{\operatorname{Cov}(X, Y) /\|X\|_{2}\|Y\|_{2}: X \in L_{2}(\mathcal{A}), Y \in L_{2}(\mathcal{B})\right\} .
$$

For the stationary sequence of random variables $\left(\xi_{k}\right)_{k \in Z}$, denote by $\mathcal{F}_{0}$ the past $\sigma$-field generated by $\xi_{k}$ with indices $k \leq 0$ and by $\mathcal{F}^{n}$ the future $\sigma$-field after $n$-steps generated by $\xi_{j}$ with indices $j \geq n$. The sequence of coefficients $\left(\rho_{n}\right)_{n \geq 1}$ is then defined by

$$
\rho_{n}=\rho\left(\mathcal{F}_{0}, \mathcal{F}^{n}\right) \text {. }
$$

If $\rho_{n}<1$ for some $n>1$, then the tail sigma field $\mathcal{F}_{-\infty}=\bigcap_{n \in Z} \sigma\left(\left(\xi_{j}\right)_{j \leq n}\right)$ is trivial; see Proposition (5.6) in Bradley [3]. In this case it is customary to say that $\mathcal{F}_{0}$ and $\mathcal{F}^{n}$ are not parallel. Now we take a random field with columns $\bar{\xi}_{j}=\left(\xi_{k, j}\right)_{k \in Z}$ independent copies of a stationary sequence with $\rho_{n}<1$ for some $n>1$. Clearly, because the columns are independent the sigma 
field $\mathcal{F}_{0,-\infty}$ is trivial. Furthermore, we shall argue that $\mathcal{F}_{-\infty, 0}$ is also trivial. To prove it, we apply Theorem 6.2 in in Csáki and Fischer [6] (see also Theorem 6.1 in Bradley [3]). According to this theorem

$$
\begin{aligned}
& \rho\left(\sigma\left(\left(\xi_{k, j}\right)_{j \in Z, k \leq 0}\right), \sigma\left(\left(\xi_{k, j}\right)_{j \in Z, k \geq n}\right)\right) \\
& \quad=\sup _{j} \rho\left(\sigma\left(\left(\xi_{k, j}\right)_{k \leq 0}\right), \sigma\left(\left(\xi_{k, j}\right)_{k \geq n}\right)\right)=\rho_{n}<1 .
\end{aligned}
$$

Therefore we also have that $\mathcal{F}_{-\infty, 0}$ is trivial and our theorem applies. For this case, we obtain the following corollary:

Corollary 7. Assume that the random field $\left(\xi_{k, j}\right)_{k, j \in Z}$ consists of columns, which are independent copies of a stationary sequence $\left(\xi_{j}\right)_{j \in Z}$ having $\rho_{n}<1$ for some $n>1$. Construct $\left(X_{\mathbf{n}}\right)_{\mathbf{n} \in Z^{2}}$ by (8) and assume that the variables are centered and square integrable. Then the results of Theorems 1 and 4 hold.

As a particular example we can take, as generator of the commuting sigma algebras, independent copies of a Gaussian sequence with a special type of spectral density. It is convenient to define the spectral density on the unit circle in the complex plane, denoted by $T$. Let $\mu$ denote normalized Lebesgue measure on $T$ (normalized so that $\mu(T)=1$ ). For a given random sequence $X:=\left(X_{k}\right)_{k \in Z}$, a "spectral density function" (if one exists) can also be viewed as a real, nonnegative, Borel, integrable function $f: T \rightarrow[0, \infty)$ such that for every $k \in Z$

$$
\operatorname{cov}\left(X_{k}, X_{0}\right)=\int_{t \in T} t^{k} f(t) \mu(\mathrm{d} t) .
$$

Let $\left(\xi_{j}\right)_{j \in Z}$ be a stationary Gaussian sequence and let $n$ be a positive integer. The following two conditions are equivalent:

(a) $\rho_{n}<1$.

(b) $\left(\xi_{j}\right)_{j \in Z}$ has a spectral density function $f$ (on $T$ ) of the form

$$
f(t)=|p(t)| \exp (u(t)+\tilde{v}(t)), \quad t \in T,
$$

where $p$ is a polynomial of degree at most $n-1$ (constant if $n=1$ ), $u$ and $v$ are real bounded Borel functions on $T$ with $\|v\|_{\infty}<\pi / 2$, and $\tilde{v}$ is the conjugate function of $v$.

For $n=1$, this equivalence is due to Helson and Szegö [17]. For general $n \geq 1$, it is due to Helson and Sarason [16], Theorem 6.

\section{Functions of i.i.d.}

Our results also hold for any random field which is Bernoulli, that is, a function of i.i.d. random field. For instance, if $\left(\xi_{\mathbf{n}}\right)_{\mathbf{n} \in Z^{d}}$ is a random field of independent, identically distributed random 
variables and we define $\left(X_{\mathbf{k}}\right)_{\mathbf{k} \in Z^{d}}$ and $\left(\mathcal{F}_{\mathbf{k}}\right)_{\mathbf{k} \in Z^{d}}$ as in Theorem 6. Then the filtration is commuting and the regularity conditions of Theorem 6 are satisfied provided the variables are centered. If in addition $X_{\mathbf{0}}$ is square integrable, then the result of Theorem 6 holds.

For the next two examples the only conditions imposed are equivalent to the existence of the fields involved.

Example 8 (Linear field). Let $\left(\xi_{\mathbf{n}}\right)_{\mathbf{n} \in Z^{d}}$ be a random field of independent, identically distributed random variables which are centered and have finite second moment. Define

$$
X_{\mathbf{k}}=\sum_{\mathbf{j} \in Z^{d}} a_{\mathbf{k}-\mathbf{j}} \xi_{\mathbf{j}}
$$

Assume that $\sum_{\mathbf{j} \in Z^{d}} a_{\mathbf{j}}^{2}<\infty$. Then the CLT in Theorem 6 holds.

Another class of nonlinear random fields is the Volterra process, which plays an important role in the nonlinear system theory.

Example 9 (Volterra field). Let $\left(\xi_{\mathbf{n}}\right)_{\mathbf{n} \in Z^{d}}$ be a random field of independent random variables identically distributed centered and with finite second moment. Define

$$
X_{\mathbf{k}}=\sum_{\mathbf{u}, \mathbf{v} \in Z^{d}} a_{\mathbf{u}, \mathbf{v}} \xi_{\mathbf{k}-\mathbf{u}} \xi_{\mathbf{k}-\mathbf{v}}
$$

where $a_{\mathbf{u}, \mathbf{v}}$ are real coefficients with $a_{\mathbf{u}, \mathbf{u}}=0$ and $\sum_{\mathbf{u}, \mathbf{v} \in Z^{d}} a_{\mathbf{u}, \mathbf{v}}^{2}<\infty$. Then the CLT in Theorem 6 holds.

\section{Supplementary results}

In this section, we prove two auxiliary results. They are laws of large numbers which have interest in themselves.

The following lemma is an extension of a result in Zhang [32].

Lemma 10. Assume that the triangular array $\left(X_{n_{2}, k}\right)_{k \in Z}$ is row-wise stationary and $\left(X_{n_{2}, k}\right)_{n_{2} \geq 1}$ is uniformly integrable for any $k$ fixed. In addition assume that $X_{n_{2}, k} \Rightarrow X_{k}$, where $X_{k}$ 's have the same distribution and are in $L_{1}$. Then, for $\lambda$-almost all $t \in[-\pi, \pi)$,

$$
\frac{1}{n_{1}} \sum_{k=1}^{n_{1}} e^{\mathrm{i} k t} X_{n_{2}, k} \rightarrow 0 \quad \text { a.s. when } n_{1} \wedge n_{2} \rightarrow \infty .
$$

Proof. Let $m \geq 1$ be a fixed integer and define consecutive blocks of indexes of size $m, I_{j}(m)=$ $\{(j-1) m+1, \ldots, m j\}$. In the set of integers from 1 to $n_{1}$ we have $\left[n_{1} / m\right]$ such blocks of 
integers and a last one containing less than $m$ indexes. By the stationarity of $\left(X_{n_{2}, k}\right)_{k \in Z}$ we have

$$
\begin{aligned}
\frac{1}{n_{1}} E\left|\sum_{k=1}^{n_{1}} e^{\mathrm{i} k t} X_{n_{2}, k}\right| \leq & \frac{1}{n_{1}} \sum_{j=1}^{\left[n_{1} / m\right]} E\left|\sum_{k \in I_{j}(m)} e^{\mathrm{i} k t} X_{n_{2}, k}\right| \\
& +\frac{1}{n_{1}} E\left|\sum_{j=\left[n_{1} / m\right] m+1}^{n_{1}} e^{\mathrm{i} j t} X_{n_{2}, j}\right| \\
\leq & \frac{1}{m} E\left|\sum_{k=1}^{m} e^{\mathrm{i} k t} X_{n_{2}, k}\right|+o_{n_{1}}(1) \quad \text { as } n_{1} \rightarrow \infty
\end{aligned}
$$

Now, again by the uniform integrability of $\left(X_{n_{2}, k}\right)_{n_{2} \geq 1}$ and the convergence of moments associated to the weak convergence (see Theorem 25.12 in Billingsley [2]) we have

$$
\begin{aligned}
& \lim _{n_{2} \rightarrow \infty} E\left|\sum_{k=1}^{m} e^{\mathrm{i} k t} X_{n_{2}, k}\right| \\
& \leq \lim _{n_{2} \rightarrow \infty} E\left|\sum_{k=1}^{m} X_{n_{2}, k} \sin k t\right|+\lim _{n_{2} \rightarrow \infty} E\left|\sum_{k=1}^{m} X_{n_{2}, k} \cos k t\right| \\
& =E\left|\sum_{k=1}^{m} X_{k} \sin k t\right|+E\left|\sum_{k=1}^{m} X_{k} \cos k t\right| .
\end{aligned}
$$

Since the $X_{k}$ 's have the same distribution, by Zhang [32], for almost all $t \in[-\pi, \pi$ )

$$
\frac{1}{m} \sum_{k=1}^{m} X_{k} \sin k t \rightarrow 0 \quad \text { and } \quad \frac{1}{m} \sum_{k=1}^{m} X_{k} \cos k t \rightarrow 0 \quad P \text {-a.s. and in } L_{1} .
$$

Lemma 11. Assume that the triangular array $\left(X_{n_{2}, k}\right)_{k \in Z}$ is row-wise stationary, mean 0 and $\left(X_{n_{2}, k}\right)_{n_{2} \geq 1}$ is uniformly integrable for any $k$ fixed. In addition assume that the finite dimensional distributions of $\left(X_{n_{2}, k}\right)_{k}$ converge in distribution to those of $\left(X_{k}\right)_{k}$ as $n_{2} \rightarrow \infty$, where $\left(X_{k}\right)_{k}$ is stationary and ergodic and in $L_{1}$. Then

$$
\frac{1}{n_{1}} \sum_{k=1}^{n_{1}} X_{n_{2}, k} \text { converges in } L_{1} \text { to } 0 \text { when } n_{1} \wedge n_{2} \rightarrow \infty .
$$

Proof. As in the previous lemma, we make blocks of variables as before and use the inequality

$$
\frac{1}{n_{1}} E\left|\sum_{k=1}^{n_{1}} X_{n_{2}, k}\right| \leq \frac{1}{m} E\left|\sum_{k=1}^{m} X_{n_{2}, k}\right|+o_{n_{1}}(1) \quad \text { as } n_{1} \rightarrow \infty .
$$


Now, by the uniform integrability and the weak convergence of the sequence $\left(X_{n_{2}, k}\right)_{n_{2} \geq 1}$, we obtain

$$
\lim _{n_{2} \rightarrow \infty} E\left|\sum_{k=1}^{m} X_{n_{2}, k}\right|=E\left|\sum_{k=1}^{m} X_{k}\right| .
$$

Furthermore, note also that by the conditions of this lemma we also have $E\left(X_{k}\right)=0$ for all $k$. By the ergodic theorem $\sum_{k=1}^{m} X_{k} / m \rightarrow 0$ a.s. and in $L_{1}$ and therefore

$$
E\left|\frac{1}{m} \sum_{k=1}^{m} X_{k}\right| \rightarrow 0 \quad \text { as } m \rightarrow \infty .
$$

The following lemma follows by Problem 34.11 in Billingsley [2].

Lemma 12. Assume that $X, Y, Z$ are integrable random variables. Then the following are equivalent

$$
\begin{array}{ll}
E(g(X, Y) \mid \sigma(Y, Z))=E(g(X, Y) \mid Y) & \text { a.s. } \\
E(g(Z, Y) \mid \sigma(Y, X))=E(g(Z, Y) \mid Y) & \text { a.s. }
\end{array}
$$

We recall the following lemma which is not difficult to verify. Its proof is left to the reader.

Lemma 13. Assume that $X, Y, Z$ are integrable random variables such that $(X, Y)$ and $Z$ are independent. Assume that $g(X, Y)$ is integrable. Then

$$
E(g(X, Y) \mid \sigma(Y, Z))=E(g(X, Y) \mid Y) \quad \text { a.s. }
$$

\section{Acknowledgements}

This research was supported in part by the NSF grant DMS-1512936. The authors are grateful to the referees for numerous suggestions, which contributed to a significant improvement of a previous version of the paper.

\section{References}

[1] Bary, N.K. (1964). A Treatise on Trigonometric Series, Vols. I, II. New York: The Macmillan Co. MR0171116

[2] Billingsley, P. (1995). Probability and Measure, 3rd ed. Wiley Series in Probability and Mathematical Statistics. New York: Wiley. MR1324786

[3] Bradley, R.C. (2007). Introduction to Strong Mixing Conditions, Vol. 2. Heber City, UT: Kendrick Press. MR2325295

[4] Carleson, L. (1966). On convergence and growth of partial sums of Fourier series. Acta Math. 116 135-157. MR0199631 
[5] Cohen, G. and Conze, J.-P. (2013). The CLT for rotated ergodic sums and related processes. Discrete Contin. Dyn. Syst. 33 3981-4002. MR3038049

[6] Csáki, P. and Fischer, J. (1963). On the general notion of maximal correlation. Magyar Tud. Akad. Mat. Kutató Int. Közl. 8 27-51. MR0166833

[7] Cuny, C., Dedecker, J. and Volný, D. (2015). A functional central limit theorem for fields of commuting transformations via martingale approximation. Zap. Nauč. Semin. POMI 441 and J. Math. Sci. (N. Y.) (2016) 219 765-781.

[8] El Machkouri, M., Volný, D. and Wu, W.B. (2013). A central limit theorem for stationary random fields. Stochastic Process. Appl. 123 1-14. MR2988107

[9] Fefferman, C. (1971). On the convergence of multiple Fourier series. Bull. Amer. Math. Soc. 77 744745.

[10] Fefferman, C. (1971). On the divergence of multiple Fourier series. Bull. Amer. Math. Soc. 77 191195. MR0279529

[11] Francos, J.M., Meiri, A.Z. and Porat, B. (1995). A Wold-like decomposition of two-dimensional discrete homogeneous random fields. Ann. Appl. Probab. 5 248-260.

[12] Gänssler, P. and Häusler, E. (1979). Remarks on the functional central limit theorem for martingales. Z. Wahrsch. Verw. Gebiete 50 237-243. MR0554543

[13] Giraudo, D. (2017). Invariance principle via orthomartingale approximation. Preprint. Available at arXiv: 1702.08288.

[14] Gordin, M.I. (2009). Martingale-coboundary representation for a class of stationary random fields. Zap. Nauchn. Sem. S.-Peterburg. Otdel. Mat. Inst. Steklov. (POMI) 364 88-108, 236. J. Math. Sci. (N. Y.) (2009) $163363-374$.

[15] Helson, H. and Lowdenslager, D. (1961). Prediction theory and Fourier series in several variables. II. Acta Math. 106 175-213. MR0176287

[16] Helson, H. and Sarason, D. (1967). Past and future. Math. Scand. 21 5-16.

[17] Helson, H. and Szegö, G. (1960). A problem in prediction theory. Ann. Mat. Pura Appl. 51 107-138.

[18] Hunt, R.I. and Young, W.S. (1974). A weighted norm inequality for Fourier series. Bull. Amer. Math. Soc. 80 274-277.

[19] Jessen, B., Marcinkiewicz, J. and Zygmund, A. (1935). Note on the differentiability of multiple integrals. Fund. Math. 25 217-234.

[20] Kallianpur, G., Miamee, A.G. and Niemi, H. (1990). On the prediction theory of two-parameter stationary random fields. J. Multivariate Anal. 32 120-149.

[21] Lifshits, M.A. and Peligrad, M. (2015). On the spectral density of stationary processes and random fields. Zap. Nauchn. Sem. S.-Peterburg. Otdel. Mat. Inst. Steklov. (POMI) 441 274-285. J. Math. Sci. (N. Y.) (2016) 219 789-797. MR3504510

[22] Marcinkiewicz, J. and Zygmund, A. (1939). On the summability of double Fourier series. Fund. Math. 32 122-132.

[23] Miller, C. (1995). A CLT for the periodograms of a $\rho^{*}$-mixing random field. Stochastic Process. Appl. 60 313-330.

[24] Peligrad, M. and Wu, W.B. (2010). Central limit theorem for Fourier transforms of stationary processes. Ann. Probab. 38 2009-2022.

[25] Peligrad, M. and Zhang, N. (2017). On the normal approximation for random fields via martingale methods. Stochastic Process. Appl. In press. DOI:10.1016/j.spa.2017.07.012.

[26] Rosenblatt, M. (1972). Central limit theorem for stationary processes. In Berkeley Symp. on Math. Statist. and Prob. Proc. Sixth Berkeley Symp. on Math. Statist. and Prob., Vol. 2 (Univ. of Calif. Press) 551-561. MR0402869

[27] Schuster, A. (1898). On the investigation of hidden periodicities with application to a supposed 26 day period of meteorological phenomena. Terr. Magn. Atmos. Electr. 3 13-41. 
[28] Volný, D. (2015). A central limit theorem for fields of martingale differences. C. R. Math. Acad. Sci. Paris 353 1159-1163. MR3427925

[29] Volný, D. and Wang, Y. (2014). An invariance principle for stationary random fields under Hannan's condition. Stochastic Process. Appl. 124 4012-4029.

[30] Wang, Y. and Woodroofe, M. (2013). A new criteria for the invariance principle for stationary random fields. Statist. Sinica 23 1673-1696.

[31] Weisz, F. (2012). Summability of multi-dimensional trigonometric Fourier series. Surv. Approx. Theory 7 1-179.

[32] Zhang, N. (2017). On the law of large numbers for discrete Fourier transform. Statist. Probab. Lett. 120 101-107. MR3567927

Received April 2017 and revised October 2017 